\title{
GENERALIZED EIGENVECTORS AND SEPARATION OF VARIABLES $\left({ }^{1}\right)$
}

\author{
BY \\ MAURICE MACHOVER
}

1. Introduction. When we apply the method of separation of variables to solve an equation like the reduced wave equation, or Helmholtz equation, $\left(\nabla^{2}+k^{2}\right) u(x, y)=f(x, y)$, we assume the solution expanded in a series of eigenfunctions of one of the variables. The coefficients depend upon the other variable. We substitute the expansion into the equation, thereby obtaining ordinary differential equations for the coefficients. The method relies upon the completeness of the eigenfunctions corresponding to one of the variables. For selfadjoint problems this assumption is justified by the usual expansion and completeness theorems of Sturm-Liouville theory [1]. For non-selfadjoint problems it is sometimes not true. An example of the latter type is the problem (for a rectangle)

$$
\begin{aligned}
\nabla^{2} u+k^{2} u & =\frac{1}{2 k_{0}} x \cos k_{0} x, \\
u(0, y) & =u(x, 0)=u(x, 1)=0, \\
u_{x}(1, y) & =c u(1, y),
\end{aligned}
$$

where $k_{0} \neq 0$ is a solution of $k_{0}=\sin k_{0} \cos k_{0}$ and $c=\cos ^{2} k_{0}$. Here the method breaks down because of the incompleteness of the eigenfunctions of the $x$ problem [6]. An attempt is made in this paper to extend the applicability of the method of separation of variables by including generalized eigenfunctions for the purpose of completion. This is modelled after the finitedimensional case. Here a basis of eigenvectors and generalized eigenvectors can be found for non-selfadjoint operators, leading to the Jordan canonical form. Some work has been done on developing expansion theorems in terms of generalized eigenfunctions. It has not been applied, however, to separation of variables. George Birkhoff proved [2] that a large class of functions could be expanded in a series of functions associated with a contour integral. They arise as residues of an integral in the complex plane of the Green's function for a linear homogeneous ordinary differential equation with "regular" boundary conditions. He did not investigate the properties of these residues in the non-selfadjoint case. J. Tamarkin [3] went further and showed that

Received by the editors April 19, 1963 and, in revised form, September 11, 1963.

( $\left.{ }^{1}\right)$ The research in this paper was supported by the United States Air Force Office of Scientific Research, under Grant No. AFOSR 62-108. Reproduction in whole or in part is permitted for any purpose of the U.S. Government. 
these residues were principal functions (generalized eigenfunctions) of the associated differential operator. He did not investigate their properties, nor those of the coefficient functions in the expansion, any further. Naimark [4], showed that for a special class of second-order equations these principal functions were generalized eigenfunctions and satisfied certain orthogonality relations with the coefficient functions. Friedman [6] pointed out that spectral representations of both ordinary and partial differential operators are modified by the presence of multiple poles in the Green's functions. He compared the generalized eigenvectors of an operator with those of its adjoint. J. Schwartz has treated more general operators and has shown that a function may be expanded in a series of eigenvectors, with possibly a finite number of generalized eigenvectors, of a spectral operator. H. P. Kramer [11] specifically studied even order differential operators and showed that they were spectral if their coefficients were bounded operators.

2. Properties of generalized eigenvectors. Let $L$ be a linear operator with domain a linear manifold $D$ in a Hilbert space $H$. We call $x$ a generalized eigenvector of rank $r$ for the operator $L$ and eigenvalue $\lambda$ when $x,(L-\lambda) x$, $\cdots,(L-\lambda)^{r-1} x$ all belong to $D$, and $(L-\lambda)^{r} x=0$ but $(L-\lambda)^{r-1} x \neq 0$. Thus let $L$ be the ordinary differential operator

$$
L x=p_{0}(t) x^{(n)}+p_{1}(t) x^{(n-1)}+\cdots+p_{n}(t) x, \quad p_{0}(t) \neq 0 \text { on }[a, b],
$$

and $D$ the linear manifold of those functions $x(t)$ with continuous derivatives up to order $n$ on $[a, b]$ such that

$$
U x=0 .
$$

Here $p_{j}(t)$ are complex-valued functions in $c^{n-j}[a, b]$, and the boundary form $U x$ is the vector

$$
U_{i} x=\sum_{j=1}^{n}\left(a_{i j} x^{(j-1)}(a)+b_{i j} x^{(j-1)}(b)\right), \quad i=1, \cdots, n
$$

(here $\left(a_{i j}\right)$ and $\left(b_{i j}\right)$ are complex matrices). Then a generalized eigenfunction of rank $r$ for $L$ and $\lambda$ is any solution of

$$
\begin{aligned}
(L-\lambda)^{r} x=0, & (L-\lambda)^{r-1} x \neq 0, \\
U x=0, & U(L-\lambda) x=0, \cdots, U(L-\lambda)^{r-1} x=0 .
\end{aligned}
$$

The notation is taken from Coddington and Levinson [5]. We see that for $x$ to lie in the domain of $L$ it must satisfy $n$ linear homogeneous boundary conditions at both end points on its first $n-1$ derivatives.

Some easily shown properties of generalized eigenvectors (not necessarily of ordinary differential operators) follow. If $x$ is of rank $r$ for $L$ and $\lambda$ then $x,(L-\lambda) x, \cdots,(L-\lambda)^{r-1} x$ form a chain of linearly independent generalized eigenvectors of decreasing rank. Of course $(L-\lambda)^{r-1} x$ is an ordinary eigenvector (rank 1). Since two chains may merge at some lower ranked com- 
ponent, we call a collection of chains "independent" when their rank one components form a linearly independent set of vectors. All the generalized eigenvectors in an independent set of chains constitute a linearly independent set of vectors. Adding a lower rank to a generalized eigenvector does not change rank, nor will the chains generated by such vectors be independent. Suppose a generalized eigenvector of rank $k$ is linearly independent from all the generalized eigenvectors of rank $k$ or less in an independent set of chains for $L$ and $\lambda$. Then it is linearly independent from all the vectors in the set. If for each eigenvalue of a finite set for $L$ we have an independent set of chains, then the totality of all the vectors is a linearly independent set. Most of these properties are known [6]. They are generalizations of properties of ordinary eigenvectors, and are proven by applying powers of $L-\lambda$ to combinations of generalized eigenvectors, and equating coefficients. Also, since the set of generalized eigenvectors of rank $m$ or less for $L$ and $\lambda$ is an invariant subspace of $H$ (for $L x=(L-\lambda) x+\lambda x$ ), then the usual theorem on the Jordan canonical form may be phrased as follows: If the space of generalized eigenvectors of rank $m$ or less for $L$ and $\lambda$ is finite-dimensional, then there exists a basis for this space consisting of independent chains. Furthermore, the number and lengths of these chains are unique.

Since we are going to consider expansion theorems in terms of generalized eigenfunctions of ordinary differential operators such as (2.1), the concept of the adjoint operator, introduced in general situations by Birkhoff [2], is essential. This is true also in the case of ordinary eigenvectors. The salient points are [5] few. First, the operator $M$, with domain the set of functions $y(t) \in C^{n}[a, b]$ satisfying $V y=0$, is said to be adjoint to $L$ if $\langle L x, y\rangle=\langle x, M y\rangle$ for all $x, y \in C^{n}[a, b]$ such that $U x=0, V y=0$. Here $V$ is a boundary form, and the scalar product is the standard formula $\langle x, y\rangle=\int_{a}^{b} x(t) \bar{y}(t) d t . L$ and $U$ determine $M$, along with its domain, uniquely. The formal adjoint $M$ is called $L^{+}$, and is the differential operator

$$
\begin{aligned}
L^{+} y=(-1)^{n}\left(\bar{p}_{0} y\right)^{(n)}+(-1)^{n-1}\left(\bar{p}_{1} y\right)^{(n-1)}+ & \cdots+\bar{p}_{n} y, \\
& p_{0}(t) \neq 0 \text { on }[a, b] .
\end{aligned}
$$

$V$ is called $U^{+}$, so that the domain of $L^{+}$is the set of functions $y(t) \in C^{n}[a, b]$ satisfying

$$
U^{+} y=0 .
$$

The problem $L^{+} y=0, U^{+} y=0$ is said to be adjoint to the problem $L x=0$, $U x=0$. The basic (alternative) theorem about adjoints is that both of these problems have the same number of linearly independent solutions; $L x=f$, $U x=0$ has a solution if and only if $f$ is orthogonal to the null space of $L^{+}[5]$. The generalized eigenfunctions of rank $m$ or less for $L$ and $\lambda$ are the solutions of

$$
(L-\lambda)^{m} x=0, \quad U x=0, \quad U(L-\lambda) x=0, \cdots, U(L-\lambda)^{m-1} x=0,
$$


whereas for $L^{+}$and $\bar{\lambda}$ they are the solutions of

$$
\begin{gathered}
\left(L^{+}-\bar{\lambda}\right)^{m} y=0, \quad U^{+} y=0, \\
U^{+}\left(L^{+}-\bar{\lambda}\right) y=0, \cdots, U^{+}\left(L^{+}-\bar{\lambda}\right)^{m-1} y=0 .
\end{gathered}
$$

Note that the solution spaces of (2.5) and (2.6) are finite-dimensional. A problem is said to be selfadjoint if $L=L^{+}$and both operators have the same domain. For such problems all generalized eigenfunctions are of rank one. The fact that they form a complete set is given by Sturm-Liouville theory. One of the othogonality properties from this theory is now generalized to higher ranks for the non-selfadjoint case.

TheOREM 2.1. If $x$ is a generalized eigenfunction for $L$ and $\lambda$, and $y$ is one for $L^{+}$and $\bar{\mu}, \mu \neq \lambda$, then $(x, y)=0$.

THEOREM 2.2. The basis of independent chains for the space of generalized eigenfunctions of rank $m$ or less for $L^{+}$and $\bar{\lambda}$ matches that for $L$ and $\lambda$ in number and lengths of chains.

Theorem 2.1 is established by induction on the ranks of $x$ and $y$. Theorem 2.2 is established by showing that the vectors of rank $j$ or less, $j=1, \cdots, m$ in each basis are the solutions of adjoint problems and thus agree in number. For details see [10]. These theorems would also follow from J. Schwartz's results [9] if it were established that $L$ is a regular differential operator.

3. The use of generalized eigenfunctions in expansion theorems. Generalized eigenfunctions are brought into expansion theorems by use of Birkhoff's result on the contour integral of the Green's function. It will be recalled [5] that if the problem

$$
(L-\lambda) x=0, \quad U x=0 \quad \text { (where } L \text { is given by (2.1)) }
$$

has only the trivial solution for at least one complex value of $\lambda$, then the Green's function $G\left(t, t^{\prime}, \lambda\right)$ for this problem exists. $G$ satisfies

$$
(L-\lambda) G=\delta\left(t-t^{\prime}\right), \quad U G=0 \quad\left(\delta\left(t-t^{\prime}\right)\right. \text { is the Dirac delta function) }
$$

for $\lambda$ not an eigenvalue, and is a meromorphic function of $\lambda$ with poles only at eigenvalues. $\int_{a}^{b} G\left(t, t^{\prime}, \lambda\right) f\left(t^{\prime}\right) d t^{\prime}$ is the unique solution of

$$
(L-\lambda) x=f, \quad U x=0,
$$

for continuous $f$, and $\lambda$ not an eigenvalue. Furthermore, the only possible limit point of the eigenvalues is $\infty$. For selfadjoint problems the eigenvalues are real and the poles of $G$ are simple. Birkhoff's result [2] is that if $U$ satisfies certain mild conditions which make the problem "regular," then for functions $f$ made up of a finite number of pieces in $[a, b]$, each real, continuous, and with a continuous derivative,

(3.2) $\frac{1}{2}[f(t+0)+f(t-0)]=\lim _{n \rightarrow \infty}\left[-\frac{1}{2 \pi i} \oint_{c_{n}} \int_{a}^{b} G\left(t, t^{\prime}, \lambda\right) f\left(t^{\prime}\right) d t^{\prime} d \lambda\right]$ on $(a, b)$. 
At $t=a$ and $t=b$ the sequence converges to linear combinations of $f(a+0)$ and $f(b-0)$. Here $C_{n}$ is a circle about the orgin of radius $n$ in the $\lambda$-plane. For selfadjoint problems the residue of $G$ at the eigenvalue $\lambda=\lambda_{p}[5]$ is

$$
-\sum_{j=m_{p}}^{n_{p}} x_{j}(t) \bar{\psi}_{j}\left(t^{\prime}\right)
$$

where the $x_{j}$ and $\psi_{j}$ are the eigenfunctions for $L$ and $L^{+}$, respectively. Thus (3.2) for this type of problem takes the form

$$
\frac{1}{2}[f(t+0)+f(t-0)]=\sum_{j=1}^{\infty} x_{j}(t)\left\langle f, \psi_{j}\right\rangle .
$$

This is the usual expansion theorem.

Thus we expect for the non-selfadjoint case that the residue of $G$ changes only by the addition of generalized eigenfunctions.

Theorem 3.1. Let $G\left(t, t^{\prime}, \lambda\right)$ be the Green's function for the problem (3.1). Then

$$
\begin{aligned}
\frac{1}{2}[f(t+0)+f(t-0)] & =\lim _{n \rightarrow \infty}\left[-\frac{1}{2 \pi i} \oint_{C_{n}} \int_{a}^{b} G\left(t, t^{\prime}, \lambda\right) f\left(t^{\prime}\right) d t^{\prime} d \lambda\right] \text { on }(a, b), \\
& =-\frac{1}{2 \pi i} \lim _{n \rightarrow \infty} \oint_{C_{n}} \int_{a}^{b} G\left(t, t^{\prime}, \lambda\right) f\left(t^{\prime}\right) d t^{\prime} d \lambda \\
& =\sum_{j=1}^{\infty} x_{j}(t)\left\langle f, \psi_{j}\right\rangle \quad \text { on }(a, b) .
\end{aligned}
$$

Here the $x_{j}(t)$ and $\psi_{j}(t)$ are generalized eigenfunctions for $L$ and $L^{+}$, respectively. Also

$$
\left\langle x_{j}, \psi_{k}\right\rangle=\delta_{j k} .
$$

More specifically, if $l$ is a pole of order $m$ of $G$, then the residue at $l$ is

$$
\begin{aligned}
\operatorname{Res}_{l}= & -\left[x_{11}(t) \bar{\psi}_{1 n_{1}}\left(t^{\prime}\right)+\cdots+x_{1 n_{1}}(t) \bar{\psi}_{11}\left(t^{\prime}\right)\right] \\
& -\left[x_{21}(t) \bar{\psi}_{2 n_{2}}\left(t^{\prime}\right)+\cdots+x_{2 n_{2}}(t) \bar{\psi}_{21}\left(t^{\prime}\right)\right]-\cdots \\
& -\left[x_{p 1}(t) \bar{\psi}_{p n_{p}}\left(t^{\prime}\right)+\cdots+x_{p n_{p}}(t) \bar{\psi}_{p 1}\left(t^{\prime}\right)\right], \quad m=n_{1} \geqq \cdots \geqq n_{p} .
\end{aligned}
$$

The $x_{i j}(t)$ constitute a chain basis for the space of generalized eigenfunctions of rank $m$ or less. The first subscript denotes chain number and the second denotes rank. The $\psi_{i j}(t)$ constitute a corresponding chain basis to the adjoint problem (2.6) (with $\lambda$ replaced by $l$ ), the subscripts having the same meaning. The orthogonality relation

$$
\left\langle x_{m n}, \psi_{m^{\prime} n^{\prime}}\right\rangle=\delta_{m m^{\prime}} \delta_{n, n_{m}+1-n^{\prime}}
$$

holds. 
We may prove this by writing out the Laurent expansion of $G$, applying powers of $L-\lambda$, and matching coefficients. See [10] for details. Alternatively this would follow from Theorem 3, Corollary 2 of J. Schwartz's article if it were established that $L$ satisfies the hypotheses therein.

4. Application to separation of variables for a rectangle. We attempt now to solve the Helmholtz equation for a rectangle by separation of variables. We will find that in some cases we must use the preceding theorems to obtain the necessary expansions. The problem is to solve

$$
\begin{aligned}
\left(\nabla^{2}+k^{2}\right) u(x, y) & =f(x, y), & & x_{1}<x<x_{2}, y_{1}<y<y_{2}, \\
u_{x}\left(x_{1}, y\right) & =z_{1} u\left(x_{1}, y\right), & & u_{x}\left(x_{2}, y\right)=z_{2} u\left(x_{2}, y\right), \\
u_{y}\left(x, y_{1}\right) & =I_{1} u\left(x, y_{1}\right), & & u_{y}\left(x, y_{2}\right)=I_{2} u\left(x, y_{2}\right) .
\end{aligned}
$$

The impedance constants, as well as $k$, may be complex. By the separation procedure we will obtain systems of ordinary differential equations. We will have to investigate their solvability and the uniqueness of solutions.

The separated problems arising from (4.1) are

$$
\begin{array}{lll}
X^{\prime \prime}+\alpha^{2} X=0, & X^{\prime}\left(x_{1}\right)=z_{1} X\left(x_{1}\right), & X^{\prime}\left(x_{2}\right)=z_{2} X\left(x_{2}\right), \\
Y^{\prime \prime}+\beta^{2} Y=0, & Y^{\prime}\left(y_{1}\right)=I_{1} Y\left(y_{1}\right), & Y^{\prime}\left(y_{2}\right)=I_{2} Y\left(y_{2}\right),
\end{array}
$$

where $\alpha^{2}+\beta^{2}=k^{2}$. The nonzero eigenvalues, $\alpha_{n}^{2}$, are the squares of the solutions of

(4.3) $\omega\left(\alpha_{n}\right) \equiv\left(z_{1}-i \alpha_{n}\right)\left(z_{2}+i \alpha_{n}\right) e^{-i \alpha_{n}\left(x_{2}-x_{1}\right)}-\left(z_{1}+i \alpha_{n}\right)\left(z_{2}-i \alpha_{n}\right) e^{i \alpha_{n}\left(x_{2}-x_{1}\right)}=0$.

Zero is an eigenvalue if and only if $z_{2}\left(z_{1} x_{1}-1\right)=z_{1}\left(z_{2} x_{2}-1\right)$, and then the corresponding eigenfunction is

$$
X_{0}=z_{1}\left(x-x_{1}\right)+1 \text {. }
$$

An application of Rouché's theorem [7] shows that the $\alpha_{n}$ asymptotically tend to $n \pi /\left(x_{2}-x_{1}\right)$ as $n$ approaches infinity. The adjoint problems of (3.2) are

$$
\begin{array}{lll}
X^{+^{\prime \prime}}+\bar{\alpha}^{2} X^{+}=0, & X^{+^{\prime}}\left(x_{1}\right)=\bar{z}_{1} X^{+}\left(x_{1}\right), & X^{+^{\prime}}\left(x_{2}\right)=\bar{z}_{2} X^{+}\left(x_{2}\right), \\
Y^{+^{\prime \prime}}+\bar{\beta}^{2} Y^{+}=0, & Y^{+^{\prime}}\left(y_{1}\right)=\bar{I}_{1} Y^{+}\left(y_{1}\right), & Y^{+^{\prime}}\left(y_{2}\right)=\bar{I}_{2} Y^{+}\left(y_{2}\right) .
\end{array}
$$

We denote by $X_{n m}$ that generalized eigenfunction corresponding to $\alpha_{n}^{2}$ which is of rank $m$. Thus for each $n$ we have a chain basis

$$
X_{n 1}, X_{n 2}, \cdots, X_{n m_{n}}
$$

of (4.2), where $m_{n}$ is the order of $\alpha_{n}$ as a pole of $G\left(x, x^{\prime}, \alpha\right)$, and correspondingly

$$
X_{n 1}^{+} X_{n 2}^{+}, \cdots, X_{n m_{n}}^{+}
$$

of (4.4). These bases satisfy

$$
\left\langle X_{n m}, X_{p q}^{+}\right\rangle=\delta_{n p} \delta_{m, m_{n}+1-q}
$$

Let $\beta_{n}^{2}=k^{2}-\alpha_{n}^{2}$ 
Having determined the bases of the $x$ problem and its adjoint by the methods of $\S 3$, we proceed according to standard technique. Thus let $u(x, y)$ be a solution of (4.1). Then, expanding $u$ and $f$ in series of the $x$ functions (we note that the $x$ problem has impedance boundary conditions, and is thus regular in the sense of Birkhoff) we get

$$
\begin{aligned}
& u(x, y)=\sum_{n=1}^{\infty} \sum_{m=1}^{m_{n}} a_{n m}(y) X_{n m}(x), \\
& f(x, y)=\sum_{n=1}^{\infty} \sum_{m=1}^{m_{n}} c_{n m}(y) X_{n m}(x)
\end{aligned}
$$

By (3.5)

$$
c_{n m}(y)=\left\langle f, X_{n, m_{n}+1-m}^{+}\right\rangle .
$$

TheOREM 3.1. Suppose (4.1) has a solution. Substitution of (4.6) into (4.1) leads to systems of ordinary differential equations for the $a_{n m}(y)$. If $k^{2}$ is not an eigenvalue of (4.1) these systems are uniquely solvable. If $k^{2}$ is an eigenvalue of (4.1) these systems are solvable if and only if $f$ is orthogonal to all the eigenfunctions of the adjoint of (4.1). The arbitrariness in the solutions of these systems induces an arbitrariness in the solution of (4.1) to within at most an eigenfunction. In either case, once the $a_{n m}(y)$ are found, the solution to (4.1) is given by (4.6).

Proof. Substitution of (4.6) into (4.1) and use of (4.5) leads to the following systems of ordinary differential equations for the $a_{n m}$ 's.

$$
\begin{aligned}
& a_{n m_{n}}^{\prime \prime}+\beta_{n}^{2} a_{n m_{n}}=c_{n m_{n}}, \\
& a_{n, m_{n}-1}^{\prime \prime}+\beta_{n}^{2} a_{n, m_{n}-1}=c_{n, m_{n}-1}+a_{n m_{n}} \text {, } \\
& a_{n 2}^{\prime \prime}+\beta_{n}^{2} a_{n 2}=c_{n 2}+a_{n 3}, \quad n=1,2, \cdots, \\
& a_{n 1}^{\prime \prime}+\beta_{n}^{2} a_{n 1}=c_{n 1}+a_{n 2} \text {, } \\
& a_{n m}^{\prime}\left(y_{1}\right)=I_{1} a_{n m}\left(y_{1}\right) \text {, } \\
& a_{n m}^{\prime}\left(y_{2}\right)=I_{2} a_{n m}\left(y_{2}\right) \text {, } \\
& m=1,2, \cdots, m_{n} ; n=1,2, \cdots
\end{aligned}
$$

We observe that the expression (4.6) for $u$ already satisfies two of the boundary conditions in (4.1). Each equation in (4.7) is of the form

$$
a^{\prime \prime}+\beta_{n}^{2} a=g, \quad a^{\prime}\left(y_{1}\right)=I_{1} a\left(y_{1}\right), \quad a^{\prime}\left(y_{2}\right)=I_{2} a\left(y_{2}\right),
$$

where $g$ differs from equation to equation. Thus the system (3.7) are uniquely solvable if and only if

$$
a^{\prime \prime}+\beta_{n}^{2} a=0, \quad a^{\prime}\left(y_{1}\right)=I_{1} a\left(y_{1}\right), \quad a^{\prime}\left(y_{2}\right)=I_{2} a\left(y_{2}\right)
$$

has only the trivial solution. In solving (4.7) we consider separately the cases where $k^{2}$ is and is not an eigenvalue of (4.1). 
Case A. We assume $k^{2}$ is not an eigenvalue of (4.1). Then (4.8) has only the trivial solution for each $n$. For if $a_{0}(y)$ were a nontrivial solution corresponding to $n_{0}$, then $u_{0}(x, y)=X_{n_{0} 1}(x) a_{0}(y)$ would be an eigenfunction of (4.1), contradicting our assumption. We may then solve (4.7) by using the Green's function $G\left(y, y^{\prime}, \beta_{n}\right)$, which is the unique solution of

$$
\begin{aligned}
G^{\prime \prime}+\beta_{n}^{2} G= & \delta\left(y-y^{\prime}\right), \quad G_{y}\left(y_{1}, y^{\prime}, \beta_{n}\right)=I_{1} G\left(y_{1}, y^{\prime}, \beta_{n}\right), \\
& G_{y}\left(y_{2}, y^{\prime}, \beta_{n}\right)=I_{2} G\left(y_{2}, y^{\prime}, \beta_{n}\right) .
\end{aligned}
$$

By the properties of $G$ the solution of (4.7) is

$$
a_{n m}(y)=\int_{y_{1}}^{y_{2}} G\left(y, y^{\prime}, \beta_{n}\right)\left[c_{n m}\left(y^{\prime}\right)+a_{n, m+1}\left(y^{\prime}\right)\right] d y^{\prime}, \quad \begin{aligned}
& m=1,2, \cdots, m_{n}, \\
& n=1,2, \cdots
\end{aligned}
$$

Here $a_{n, m_{n}+1}=0$. The Green's function is

$$
\begin{aligned}
G\left(y, y^{\prime}, \beta_{n}\right)= & \frac{\left[I_{2} y_{>}+\left(1-I_{2} y_{2}\right)\right]\left[I_{1} y_{<}+\left(1-I_{1} y_{1}\right)\right]}{I_{2}\left(1-I_{1} y_{1}\right)-I_{1}\left(1-I_{2} y_{2}\right)} \quad \text { for } \beta_{n}=0, \\
= & \frac{-i}{2 \beta_{n} \Delta}\left[\left(I_{2}+i \beta_{n}\right) e^{i \beta_{n}\left(y_{>}-y_{2}\right)}-\left(I_{2}-i \beta_{n}\right) e^{-i \beta_{n}\left(y_{>}-y_{2}\right)}\right] \\
& \times\left[\left(I_{1}+i \beta_{n}\right) e^{i \beta_{n}\left(y_{<}-y_{1}\right)}-\left(I_{1}-i \beta_{n}\right) e^{-i \beta n\left(y_{<}-y_{1}\right)}\right] . \text { for } \beta_{n} \neq 0 .
\end{aligned}
$$

Here $y_{>}=\max \left(y, y^{\prime}\right), y_{<}=\min \left(y, y^{\prime}\right)$, and

$$
\Delta=\left(I_{1}+i \beta_{n}\right)\left(I_{2}-i \beta_{n}\right) e^{i \beta_{n}\left(y_{2}-y_{1}\right)}-\left(I_{1}-i \beta_{n}\right)\left(I_{2}+i \beta_{n}\right) e^{-i \beta_{n}\left(y_{2}-y_{1}\right)}
$$

From (4.6), (4.9), (4.10) and the form of the $X_{n m}^{+}$, the $a_{n m}(y)$ turn out to be linear combinations of functions of the form

$$
e^{ \pm i \beta_{n} y} \int_{y_{1}}^{y} \int_{x_{1}}^{x_{2}} p_{j}(x) f\left(x, y^{\prime}\right) e^{ \pm i\left|\alpha_{n}\left(x-x_{1}\right) \pm \beta_{n} y^{\prime}\right|} d x d y^{\prime} .
$$

Also from (4.6) and (4.9) the solution for $u$ is

$$
u(x, y)=\sum_{n=1}^{\infty} \sum_{m=1}^{m_{n}} \int_{y_{1}}^{y_{2}} G\left(y, y^{\prime}, \beta_{n}\right)\left[c_{n m}\left(y^{\prime}\right)+a_{n, m+1}\left(y^{\prime}\right)\right] d y^{\prime} X_{n m}(x),
$$

concluding Case A.

Case B. Now let $k^{2}$ be an eigenvalue. We show here that (4.7) is solvable if and only if $f$ is orthogonal to all the solutions of the homogeneous adjoint problem of (4.1). Furthermore any arbitrariness in the solution of (4.7) introduces an arbitrariness in $u$ to within at most an eigenfunction of (4.1). Before establishing this we must investigate the nature of the eigenfunetions of (4.1) and its adjoint problem. This adjoint problem is

$$
\begin{array}{cc}
\left(\nabla^{2}+\bar{k}^{2}\right) v=0 \\
v_{x}\left(x_{1}, y\right)=\overline{z_{1}} v\left(x_{1}, y\right), & v_{x}\left(x_{2}, y\right)=\bar{z}_{2} v\left(x_{2}, y\right), \\
v_{y}\left(x, y_{1}\right)=\bar{I}_{1} v\left(x, y_{1}\right), & v_{y}\left(x, y_{2}\right)=\bar{I}_{2} v\left(x, y_{2}\right) .
\end{array}
$$


We show this by using Green's theorem. Let $u$ and $v$ be any functions satisfying the boundary conditions (though not necessarily the differential equations) of (4.1) and (4.11), respectively. Then

$$
\begin{aligned}
\left\langle\nabla^{2} u, v\right\rangle & =\int_{y_{1}}^{y_{2}} \int_{x_{1}}^{x_{2}} \bar{v} \nabla^{2} u d x d y \\
& =\int_{y_{1}}^{y_{2}} \int_{x_{1}}^{x_{2}} u \nabla^{2} \bar{v} d x d y+\oint\left(\bar{v} \frac{\partial u}{\partial n}-u \frac{\partial \vec{v}}{\partial n}\right) d s=\left\langle u, \nabla^{2} v\right\rangle .
\end{aligned}
$$

The boundary integral vanishes by virtue of the boundary conditions.

We now investigate what the eigenfunctions of (4.1) and (4.11) are in terms of the solutions of the separated problems. The answer is given by the following.

LEMMA 4.1. If $k^{2}$ is an eigenvalue of (4.1) then $k^{2}=\alpha^{2}+\beta^{2}$ for some pair of $\alpha$ and $\beta$ (there may be more than one pair, that is, $k^{2}$ may be degenerate), where $\alpha^{2}$ and $\beta^{2}$ are eigenvalues of (4.2). We denote the chains corresponding to them by

$$
X_{1}, X_{2}, \cdots, X_{m} ; Y_{1}, Y_{2}, \cdots, Y_{n},
$$

where $m, n$ are the indices of $\alpha^{2}$ and $\beta^{2}$, respectively. Then the eigenfunctions of (4.1) are the linear combinations of

$$
\begin{gathered}
X_{1} Y_{1}, X_{1} Y_{2}-X_{2} Y_{1}, \cdots, X_{1} Y_{j-1}-X_{2} Y_{j-2}+\cdots+(-1)^{j} X_{j-1} Y_{1}, \\
X_{1} Y_{j}-X_{2} Y_{j-1}+\cdots+(-1)^{j+1} X_{j} Y_{1}, \quad j=\min (m, n) .
\end{gathered}
$$

We refer to these as chain products. (In the case of degeneracy we have such chain products for each pair of eigenvalues $\alpha_{n}^{2}, \beta_{n}^{2}$ such that $k^{2}=\alpha_{n}^{2}+\beta_{n}^{2}$.)

Proof. First let

$$
w(x, y)=X_{1} Y_{k-1}-X_{2} Y_{k-2}+\cdots+(-1)^{k} X_{k-1} Y_{1}, \quad 2 \leqq k \leqq j+1 .
$$

We will now show that $w$ is an eigenfunction of (4.1). We define the operators $L$ and $M$ by $L w(x, y)=-w_{x x}(x, y), M w(x, y)=-w_{y y}(x, y)$. Thus (calling $X_{0}=Y_{0}=0$ ) to preserve notation)

$$
\begin{aligned}
\left(\nabla^{2}+k^{2}\right) w(x, y) & =-\left(L-\alpha^{2}+M-\beta^{2}\right) \sum_{p=1}^{k-1}(-1)^{p+1} X_{p}(x) Y_{k-p}(y) \\
& =-\sum_{p=1}^{k-1}(-1)^{p+1}\left[X_{p-1}(x) Y_{k-p}(y)+X_{p}(x) Y_{k-1-p}(y)\right] \\
& =\sum_{p=2}^{k-1}(-1)^{p} X_{p-1}(x) Y_{k-p}(y)+\sum_{p=1}^{k-2}(-1)^{p} X_{p}(x) Y_{k-1-p}(y) \\
& =\sum_{p=2}^{k-1}(-1)^{p} X_{p-1}(x) Y_{k-p}(y)-\sum_{p^{\prime}=2}^{k-1}(-1)^{p^{\prime}} X_{p^{\prime}-1}(x) Y_{k-p^{\prime}}(y) \\
& =0 .
\end{aligned}
$$


Also $w$ satisfies the boundary conditions by virtue of the conditions on the $X_{p}$ and $Y_{p}$, and is thus an eigenfunction. Next we assume that $w$ is an eigenfunction of (4.1). We will find its form. From previous arguments

$$
w(x, y)=\sum_{n=1}^{\infty} \sum_{m=1}^{m_{n}} a_{n m}(y) X_{n m}(x),
$$

where the $a_{n m}$ are to be found. Substitution into (4.1) with $f=0$ leads to (4.7), with $c_{n_{1}}=c_{n_{2}}=\cdots=c_{n m_{n}}=0$.

For those $n$ for which $\beta_{n}^{2}$ is not an eigenvalue, $a_{n_{1}}=a_{n_{2}}=\cdots=a_{n m_{n}}=0$. For some $n=n_{0}, \beta_{n}^{2}$ is an eigenvalue. Otherwise $a_{n m}=0$ for all $n, m$ and $u$ would not be an eigenfunction. Let $\beta^{2}=\beta_{n_{0}}^{2} \alpha^{2}=\alpha_{n_{0}}^{2}$. Dropping the chain subscript from the $a_{n_{0} m}$ and from the generalized eigenfunctions, the $n_{0}$ th system of (4.7) becomes

$$
\begin{array}{rlr}
a_{m}^{\prime \prime}+\beta^{2} a_{m} & =0, & \\
a_{m-1}^{\prime \prime}+\beta^{2} a_{m-1} & =a_{m} & \\
\cdot \cdot \cdot & a_{p}^{\prime}\left(y_{1}\right)=I_{1} a_{p}\left(y_{1}\right), & a_{p}^{\prime}\left(y_{2}\right)=I_{2} a_{p}\left(y_{2}\right), \\
a_{2}^{\prime \prime}+\beta^{2} a_{2} & =a_{3}, & p=1,2, \cdots, m . \\
a_{1}^{\prime \prime}+\beta^{2} a_{1} & =a_{2}, &
\end{array}
$$

We solve this separately for the cases $m \leqq n$ and $m>n$.

Case a. $m \leqq n$. The general solution of the first equation is $a_{m}=c Y_{1}$. The second equation is then

$$
a_{m-1}^{\prime \prime}+\beta^{2} a_{m-1}=c Y_{1},
$$

whose general solution is $a_{m-1}=-c Y_{2}+d Y_{1}$, since $-c Y_{2}$ is a particular solution. Similarly the general solution of the third equation is $a_{m-2}=c Y_{3}-d Y_{2}$ $+e Y_{1}$. Continuing this way we arrive at the last equation, whose general solution is

$$
a_{1}=(-1)^{m-1} c Y_{m}+(-1)^{m-2} d Y_{m-1}+\cdots+(-1)^{1} i Y_{2}+(-1)^{0} j Y_{1} .
$$

Thus (assuming $\beta_{n_{0}}^{2}$ is the only $\beta_{n}^{2}$ that is an eigenvalue) we find

$$
\begin{aligned}
u(x, y)=\sum_{j=1}^{m} & a_{j}(y) X_{j}(x)=(-1)^{m-1} c\left(X_{1} Y_{m}-X_{2} Y_{m-1}+\cdots+(-1)^{m-1} X_{m} Y_{1}\right) \\
& +(-1)^{m-2} d\left(X_{1} Y_{m-1}-X_{2} Y_{m-2}+\cdots+(-1)^{m-2} X_{m-1} Y_{1}\right)+\cdots \\
& +(-1)^{0} j X_{1} Y_{1}
\end{aligned}
$$

which is of the asserted form.

Case b. $m>n$. Again the solution of the first equation is $a_{m}=c_{1} Y_{1}$. The second has solution $a_{m-1}=-c_{1} Y_{2}+c_{2} Y_{1}$, and so on, until we come to the $(n+1)$ st equation. This equation is

$$
a_{m-n}^{\prime \prime}+\beta^{2} a_{m-n}=a_{m-n+1}=(-1)^{n-1} c_{1} Y_{n}+(-1)^{n-2} c_{2} Y_{n-1}+\cdots+(-1)^{0} c_{n} Y_{1} \text {. }
$$


Because this equation has a solution, the alternative theorem implies

$$
0=\left\langle a_{m-n+1}, Y_{1}^{+}\right\rangle=\left\langle(-1)^{n-1} c_{1} Y_{n}, Y_{1}^{+}\right\rangle=(-1)^{n-1} c_{1} .
$$

Thus its general solution is

$$
a_{m-n}=(-1)^{n-1} c_{2} Y_{n}+(-1)^{n-2} c_{3} Y_{n-1}+\cdots+(-1)^{1} c_{n} Y_{2}+(-1)^{0} c_{n+1} Y_{1} .
$$

If $m-n=1$ we are finished. If not, the next equation is

$$
a_{m-n-1}^{\prime \prime}+\beta^{2} a_{m-n-1}=a_{m-n} \text {. }
$$

Again, since this has a solution we get $c_{2}=0$. We continue in this manner, using the alternative theorem. When we get to the $m$ th equation we have $c_{1}=c_{2}=\cdots=c_{m-n-1}=0$. The $m$ th equation is

$$
a_{1}^{\prime \prime}+\beta^{2} a_{1}=a_{2}=(-1)^{n-1} c_{m-n} Y_{n}+(-1)^{n-2} c_{m-n+1} Y_{n-1}+\cdots+(-1)^{0} c_{m-1} Y_{1} .
$$

Thus $c_{m-n}=0$. Its solution is

$$
a_{1}=(-1)^{n-1} c_{m-n+1} Y_{n}+(-1)^{n-2} c_{m-n+2} Y_{n-1}+\cdots+(-1)^{0} c_{m} Y_{1} .
$$

Thus since $a_{n+1}=a_{n+2}=\cdots=a_{m}=0$ we finally get

$$
\begin{aligned}
w(x, y)= & (-1)^{0} c_{m}\left(X_{1} Y_{1}\right)+(-1)^{1} c_{m-1}\left(X_{1} Y_{2}-X_{2} Y_{1}\right)+\cdots \\
& +(-1)^{n-1} c_{m-n+1}\left(X_{1} Y_{n}+\cdots+(-1)^{n-1} X_{n} Y_{1}\right) .
\end{aligned}
$$

This is of the asserted form, proving the Lemma.

Applying Lemma 4.1 to obtain the eigenfunction of (4.11) we see that they are linear combinations of

$$
\begin{gathered}
X_{1}^{+} Y_{1}^{+}, X_{1}^{+} Y_{2}^{+}-X_{2}^{+} Y_{1}^{+}, \ldots, X_{1}^{+} Y_{j-1}^{+}-X_{2}^{+} Y_{j-2}^{+}+\cdots+(-1)^{j} X_{j-1}^{+} Y_{1}^{+}, \\
X_{1}^{+} Y_{j}^{+} X_{2}^{+} Y_{j-1}^{+}+\cdots+(-1)^{j+1} X_{j}^{+} Y_{1}^{+}, \quad j=\min (m, n) .
\end{gathered}
$$

The $X_{p}^{+}$and $Y_{p}^{+}$are generalized eigenfunctions of (4.4). The orthogonality relations among these functions are

$$
\left\langle X_{i}, X_{j}^{+}\right\rangle=\delta_{i, m+1-j}, \quad\left\langle Y_{i}, Y_{j}^{+}\right\rangle=\delta_{i, n+1-j} .
$$

We are now ready to establish our initial assertion on the necessary and sufficient conditions for the solvability of (4.7) when $k^{2}$ is an eigenvalue. Dropping the chain subscript the $n_{0}$ th system of (4.7) becomes

$$
\begin{array}{rlr}
a_{m}^{\prime \prime}+\beta^{2} a_{m} & =c_{m}, & \\
a_{m-1}^{\prime \prime}+\beta^{2} a_{m-1} & =c_{m-1}+a_{m}, & \\
\cdot \cdot & \cdot & a_{p}^{\prime}\left(y_{1}\right)=I_{1} a_{p}\left(y_{1}\right), a_{p}^{\prime}\left(y_{2}\right)=I_{2} a_{p}\left(y_{2}\right), \\
a_{2}^{\prime \prime}+\beta^{2} a_{2} & =c_{2}+a_{3}, & p=1,2, \cdots, m . \\
a_{1}^{\prime \prime}+\beta^{2} a_{1} & =c_{1}+a_{2}, &
\end{array}
$$

First assume that (4.7) has a solution for the $a_{n m}$. Define 


$$
\tilde{u}(x, y)=\sum_{n=1}^{\infty} \sum_{m=1}^{m_{n}} a_{n m}(y) X_{n m}(x) .
$$

Then

$$
\left(\nabla^{2}+k^{2}\right) \tilde{u}(x, y)=f(x, y) .
$$

Let $u^{+}(x, y)$ be any solution of (4.11). Then

$$
\begin{aligned}
\int_{y_{1}}^{y_{2}} \int_{x_{1}}^{x_{2}} f(x, y) \bar{u}^{+}(x, y) d x d y & =\left\langle f, u^{+}\right\rangle=\left\langle\left(\nabla^{2}+k^{2}\right) \tilde{u}, u^{+}\right\rangle \\
& =\left\langle\tilde{u},\left(\nabla^{2}+\bar{k}^{2}\right) u^{+}\right\rangle=0,
\end{aligned}
$$

establishing the necessity. Now assume that $f$ is orthogonal to all solutions of (4.11). Those systems of (4.7) for which $\beta_{n}^{2}$ is not an eigenvalue are uniquely solvable, as before, by the method of Green's function. For the remaining systems, a typical one being (4.18), we have to show that in each equation the inhomogeneous term satisfies the orthogonality criterion of the alternative theorem. By our assumption

$$
\begin{array}{r}
\int_{y_{1}}^{y_{2}} \int_{x_{1}}^{x_{2}} f(x, y) \bar{X}_{1}^{+}(x) \bar{Y}_{1}^{+}(y) d x d y=0, \\
\int_{y_{1}}^{y_{2}} \int_{x_{1}}^{x_{2}} f(x, y) \bar{X}_{1}^{+}(x) \bar{Y}_{2}^{+}(y) d x d y-\int_{y_{1}}^{y_{2}} \int_{x_{1}}^{x_{2}} f(x, y) \bar{X}_{2}^{+}(x) \bar{Y}_{1}^{+}(y) d x d y=0,
\end{array}
$$

$$
\begin{array}{r}
\int_{y_{1}}^{y_{2}} \int_{x_{1}}^{x_{2}} f(x, y) \bar{X}_{1}^{+}(x) \bar{Y}_{j-1}^{+}(y) d x d y-\int_{y_{1}}^{y_{2}} \int_{x_{1}}^{x_{2}} f(x, y) \bar{X}_{2}^{+}(x) \bar{Y}_{j-2}^{+}(y) d x d y+\cdots \\
+(-1)^{j} \int_{y_{1}}^{y_{2}} \int_{x_{1}}^{x_{2}} f(x, y) \bar{X}_{j-1}^{+}(x) \bar{Y}_{1}^{+}(y) d x d y=0
\end{array}
$$

$$
\begin{array}{r}
\int_{y_{1}}^{y_{2}} \int_{x_{1}}^{x_{2}} f(x, y) \bar{X}_{1}^{+}(x) \bar{Y}_{j}^{+}(y) d x d y-\int_{y_{1}}^{y_{2}} \int_{x_{1}}^{x_{2}} f(x, y) \bar{X}_{2}^{+}(x) \bar{Y}_{j-1}^{+}(y) d x d y+\cdots \\
+(-1)^{j+1} \int_{y_{1}}^{y_{2}} \int_{x_{1}}^{x_{2}} f(x, y) \bar{X}_{j}^{+}(x) \bar{Y}_{1}^{+}(y) d x d y=0 .
\end{array}
$$

We want to show

$$
\left\langle c_{m}, Y_{1}^{+}\right\rangle=\left\langle c_{m-1}+a_{m}, Y_{1}^{+}\right\rangle=\cdots=\left\langle c_{1}+a_{2}, Y_{1}^{+}\right\rangle=0 .
$$

Starting with the first equation,

$$
\left\langle c_{m}, Y_{1}^{+}\right\rangle=\int_{y_{1}}^{y_{2}} \int_{x_{1}}^{x_{2}} f(x, y) \bar{X}_{1}^{+}(x) \bar{Y}_{1}^{+}(y) d x d y=0 .
$$

Thus this equation is solvable, its general solution being 


$$
a_{m}=a_{m_{p}}+d_{1} Y_{1}
$$

where $a_{m_{p}}$ is a particular solution and $d_{1}$ is arbitrary. Then

$$
\begin{aligned}
\left\langle c_{m-1}+a_{m}, Y_{1}^{+}\right\rangle & =\int_{y_{1}}^{y_{2}} \int_{x_{1}}^{x_{2}} f(x, y) \bar{X}_{2}^{+}(x) \bar{Y}_{1}^{+}(y) d x d y+\left\langle a_{m}, Y_{1}^{+}\right\rangle \\
& =\int_{y_{1}}^{y_{2}} \int_{x_{1}}^{x_{2}} f(x, y) \bar{X}_{1}^{+}(x) \bar{Y}_{2}^{+}(y) d x d y+\left\langle a_{m}, Y_{1}^{+}\right\rangle \\
& =\int_{y_{1}}^{y_{2}} c_{m}(y) \bar{Y}_{2}^{+}(y) d y+\left\langle a_{m}, Y_{1}^{+}\right\rangle \\
& =\int_{y_{1}}^{y_{2}}\left[a_{m}^{\prime \prime}(y)+\beta^{2} a_{m}(y)\right] \bar{Y}_{2}^{+}(y) d y+\left\langle a_{m}, Y_{1}^{+}\right\rangle \\
& =\left[a_{m}^{\prime} \bar{Y}_{2}^{+}-a_{m} \bar{Y}_{2}^{+}\right]_{y_{1}}^{y_{2}}-\left\langle a_{m}, Y_{1}^{+}\right\rangle+\left\langle a_{m}, Y_{1}^{+}\right\rangle=0
\end{aligned}
$$

by (4.18). The general solution of the second equation is then

$$
a_{m-1}=a_{(m-1) p}-d_{1} Y_{2}+d_{2} Y_{1},
$$

where $a_{(m-1) p}$ is any particular solution of $a^{\prime \prime}+\beta^{2} a=c_{m-1}+a_{m p}$. Note that this equation is solvable, just set $d_{1}=0$ in (4.20). We continue proving (4.20) in the same manner. We replace each $c_{p}$ by $\int_{y_{1}}^{y_{2}} \int_{x_{1}}^{x_{2}} f(x, y)$ . $\bar{X}_{m+1-p}^{+}(x) d x d y$. Then we use (4.19) to replace these expressions by ones involving lower ranked $x$ adjoint functions. We now replace these latter integrals by higher indexed $c_{q}$ 's; use (4.18) to replace these by derivatives of the higher $a_{q}^{\prime}$ 's. Then we integrate by parts, to get $\left(L^{+}-\bar{\beta}^{2}\right)$ operating on the adjoint $y$ functions. Finally we use the boundary conditions to show that the resulting expression is zero. After establishing

$$
\left\langle c_{m}, Y_{1}^{+}\right\rangle=\left\langle c_{m-1}+a_{m}, Y_{1}^{+}\right\rangle=\cdots=\left\langle c_{m-(j-1)}+a_{m-(j-2)}, Y_{1}^{+}\right\rangle=0
$$

we have for the general solution of the system of the first $j$ equations of (4.18),

$$
\begin{aligned}
a_{m} & =a_{m_{p}}+d_{1} Y_{1}, \\
a_{m-1} & =a_{(m-1) p}-d_{1} Y_{2}+d_{2} Y_{1},
\end{aligned}
$$

$$
\begin{array}{r}
a_{m-(j-2)}=a_{[m-(j-2)] p}+(-1)^{j-2} d_{1} Y_{j-1}+(-1)^{\jmath-3} d_{2} Y_{j-2}+\cdots \\
+(-1)^{1} d_{j-2} Y_{2}+d_{j-1} Y_{1}, \\
a_{m-(j-1)}=a_{[m-(j-1)] p}+(-1)^{j-1} d_{1} Y_{j}+(-1)^{\jmath-2} d_{2} Y_{j-1}+\cdots \\
+(-1)^{1} d_{j-1} Y_{2}+d_{j} Y_{1} .
\end{array}
$$

Here the $d$ 's are arbitrary and $a_{(m-q)}, q=0,1, \cdots, j-1$, is any particular solution of

$$
a^{\prime \prime}+\beta^{2} a=c_{m-q}+a_{\mid m-(q-1)\rfloor_{p}},
$$


where $a_{(m+1)_{p}}=0$. By (4.23) this equation is solvable. (Just set the $d$ 's $=0$ ). If $j=m$ we have covered all the equations of (4.18) and have established our assertion. The general solution is (4.24). Thus the contribution of this system to $u$ is

$$
\begin{array}{r}
\sum_{p=1}^{m} a_{p}(y) X_{p}(x)=d_{m}\left(X_{1} Y_{1}\right)+(-1)^{1} d_{m-1}\left(X_{1} Y_{2}-X_{2} Y_{1}\right) \\
+(-1)^{2} d_{m-2}\left(X_{1} Y_{3}-X_{2} Y_{2}+X_{3} Y_{1}\right)+\cdots \\
+(-1)^{m-1} d_{1}\left(X_{1} Y_{m}-X_{2} Y_{m-1}+\cdots+(-1)^{m} X_{m-1} Y_{2}+(-1)^{m+1} X_{m} Y_{1}\right) \\
+X_{1} a_{1_{p}}+X_{2} a_{p_{p}}+\cdots+X_{m} a_{m_{p}}
\end{array}
$$

This is arbitrary to within an eigenfunction of (4.1). If instead $j=n<m$ we have to go further and show that

$$
\begin{aligned}
\left\langle c_{m-n}+a_{m-(n-1)}, Y_{1}^{+}\right\rangle & =\left\langle c_{m-(n+1)}+a_{m-n}, Y_{1}^{+}\right\rangle=\cdots \\
& =\left\langle c_{2}+a_{3}, Y_{1}^{+}\right\rangle=\left\langle c_{1}+a_{2}, Y_{1}^{+}\right\rangle=0 .
\end{aligned}
$$

To do this we will have to fix some of the $d$ 's, thus restricting the generality of our solution.

Case a. $j=n<m=n-\quad r \leqq 2 n$, therefore $r \leqq n$.

In this case the general solution of (4.18) is

$$
\begin{aligned}
& a_{m}=a_{m_{p}}+d_{1_{0}} Y_{1} \text {, } \\
& a_{m-1}=a_{(m-1) p}+(-1)^{1} d_{1_{0}} Y_{2}+d_{2_{0}} Y_{1} \text {, } \\
& a_{m-2}=a_{(m-2) p}+(-1)^{2} d_{1_{0}} Y_{3}+(-1)^{1} d_{2_{0}} Y_{2}+d_{3_{0}} Y_{1} \text {, } \\
& a_{m-(r-1)}=a_{\mid m-(r-1) ! p}+(-1)^{r-1} d_{1_{0}} Y_{r}+(-1)^{r-2} d_{2_{0}} Y_{r-1}+\cdots \\
& +(-1)^{1} d_{(r-1)_{0}} Y_{2}+d_{r_{0}} Y_{1}, \\
& a_{m-r}=a_{(m-r)_{p}}+(-1)^{r} d_{1_{0}} Y_{r+1}+(-1)^{r-1} d_{2_{0}} Y_{r}+\cdots \\
& +(-1)^{1} d_{r_{0}} Y_{2}+d_{r+1} Y_{1} \quad(\text { delete if } r=n), \\
& a_{m-(n-1)}=a_{\mid m-(n-1) !}+(-1)^{n-1} d_{1_{0}} Y_{n}+(-1)^{n-2} d_{2_{0}} Y_{n-1}+\cdots \\
& +(-1)^{n-r} d_{r 0} Y_{n-(r-1)}+(-1)^{n-(r+1)} d_{r+1} Y_{n-r}+\cdots+(-1)^{1} d_{n-1} Y_{2}+d_{n} Y_{1} \text {, } \\
& a_{m-n}=a_{(m-n) p}+(-1)^{n-1} d_{2_{0}} Y_{n}+\cdots+(-1)^{n-(r-1)} d_{r_{0}} Y_{n-(r-2)} \\
& +(-1)^{n-r} d_{r+1} Y_{n-(r-1)}+\cdots+(-1)^{1} d_{n} Y_{2}+d_{n+1} Y_{1}, \\
& a_{2}=a_{2_{p}}+(-1)^{n-1} d_{r_{0}} Y_{n}+(-1)^{n-2} d_{r+1} Y_{n-1}+\cdots \\
& +(-1)^{1} d_{m-2} Y_{2}+d_{m-1} Y_{1}, \\
& a_{1}=a_{1_{p}}+(-1)^{n-1} d_{r+1} Y_{n}+\cdots+(-1)^{1} d_{m-1} Y_{2}+d_{m} Y_{1} \text {. }
\end{aligned}
$$


Here $d_{r+1}, d_{r+2}, \cdots, d_{m}$ are arbitrary, and $a_{1_{p}}, \ldots, a_{m_{p}}, d_{1_{0}}, \cdots, d_{r_{0}}$ are defined as follows: $a_{(m-q) p}$ for $q=0,1, \cdots, n-1$ is any particular solution of

$$
a^{\prime \prime}+\beta^{2} a=c_{m-q}+a_{\lfloor m-(q-1) ! p},
$$

where we set $a_{(m+1)_{p}}=0$. This is solvable by (4.23). We define in order $d_{1_{0}}$, $a_{(m-n) p}, d_{2_{0},} a_{[m-(n+1) !}, \cdots, d_{r_{0}}, a_{1_{p}}$ by

$$
d_{q_{0}}=-(-1)^{n-1}\left[\left\langle c m-(n+q-1), Y_{1}^{+}\right\rangle+\left\langle a_{\mid m-(n+q-2)] p}, Y_{1}^{+}\right\rangle\right],
$$

$$
\begin{array}{r}
a_{(m-q) p}^{\prime \prime}+\beta^{2} a_{(m-q) p}=c_{m-q}+a_{(m-(q-1) !}+(-1)^{n-1} d_{(q+1-n)_{0}} Y_{n}, \\
q=n, n+1, \cdots, m-2, m-1 .
\end{array}
$$

Note that the choice of $d_{1_{0}}, \cdots, d_{r_{0}}$ makes this latter equation solvable. One can now check that (4.27) holds and that (4.28) is indeed the general solution.

Case b.

$$
j=n<m=n+r>2 n, \quad \text { therefore } r>n .
$$

Again we define $a_{1_{p}}, \cdots, a_{m_{p}}, d_{1_{0}}, \cdots, d_{r_{0}}$ by (4.29) and (4.30). The general solution of (4.18) is then

$$
\begin{aligned}
& a_{m}=a_{m_{p}}+d_{1_{0}} Y_{1}, \\
& a_{m-1}=a_{(m-1) p}+(-1)^{1} d_{1_{0}} Y_{2}+d_{2_{0}} Y_{1} \text {, } \\
& a_{m-(n-1)}=a_{[m-(n-1) !}+(-1)^{n-1} d_{1_{0}} Y_{n}+(-1)^{n-2} d_{2_{0}} Y_{n-1}+\cdots \\
& +(-1)^{1} d_{(n-1)_{0}} Y_{2}+d_{n_{0}} Y_{1}, \\
& a_{m-n}=a_{(m-n) p}+(-1)^{n-1} d_{2_{0}} Y_{n}+\cdots+(-1)^{1} d_{n_{0}} Y_{2}+d_{(n+1)_{0}} Y_{1}, \\
& a_{m-(r-1)}=a_{\left[m-(r-1) l_{p}\right.}+(-1)^{n-1} d_{(r-n+1)_{0}} Y_{n}+\cdots+(-1)^{1} d_{(r-1)_{0}} Y_{2}+d_{r_{0}} Y_{1}, \\
& a_{m-r}=a_{|m-r| p}+(-1)^{n-1} d_{(r-n+2) 0} Y_{n}+\cdots+(-1)^{1} d_{r_{0}} Y_{2}+d_{r+1} Y_{1}, \\
& a_{2}=a_{2_{p}}+(-1)^{n-1} d_{r_{0}} Y_{n}+(-1)^{n-2} d_{r+1} Y_{n-1}+\cdots \\
& +(-1)^{1} d_{m-2} Y_{2}+d_{m-1} Y_{1}, \\
& a_{1}=a_{1_{p}}+(-1)^{n-1} d_{r+1} Y_{n}+(-1)^{n-2} d_{r+2} Y_{n-1}+\cdots \\
& +(-1)^{1} d_{m-1} Y_{2}+d_{m} Y_{1} .
\end{aligned}
$$

Thus in either case (4.27) holds and one can check that the contribution of (4.18) to $u$ is arbitrary to within an eigenfunction of (4.1). Thus (4.7) is solvable for the case $k^{2}$ an eigenvalue to within the asserted degree of arbitrariness. Thus, we have proved Theorem 4.1.

It is worth noting that the actual solution of (4.1) may be carried out in two steps. The first uses the unmodified procedure of separation of variables, dealing only with ordinary eigenvectors. It involves solving 


$$
\begin{array}{ll}
\left(\nabla^{2}+k^{2}\right) u_{1}(x, y)=f(x, y)-\sum_{n=1}^{\infty} \sum_{m=2}^{m_{n}} c_{n m}(y) X_{n m}(x) \\
u_{1_{x}}\left(x_{1}, y\right)=z_{1} u_{1}\left(x_{1}, y\right), & u_{1_{x}}\left(x_{2}, y\right)=z_{2} u_{1}\left(x_{2}, y\right) \\
u_{1_{y}}\left(x, y_{1}\right)=I_{1} u_{1}\left(x, y_{1}\right), & u_{1_{y}}\left(x, y_{2}\right)=I_{2} u_{1}\left(x, y_{2}\right)
\end{array}
$$

by substituting in

$$
\begin{gathered}
u_{1}(x, y)=\sum_{n=1}^{\infty} a_{n 1}(y) X_{n 1}(x) \\
f(x, y)-\sum_{n=1}^{\infty} \sum_{m=2}^{m_{n}} c_{n m}(y) X_{n m}(x)=\sum_{n=1}^{\infty} c_{n 1}(y) X_{n 1}(x) .
\end{gathered}
$$

This leads to the single equation

$$
a_{n 1}^{\prime \prime}+\beta_{n}^{2} a_{n 1}=c_{n 1}, \quad a_{n 1}^{\prime}\left(y_{1}\right)=I_{1} a_{n 1}\left(y_{1}\right), \quad a_{n 1}^{\prime}\left(y_{2}\right)=I_{2} a_{n 1}\left(y_{2}\right) .
$$

Any consideration of higher ranks in this part would only give us systems of ordinary differential equations whose solutions are

$$
a_{n m}=0 \text { for } m>1 \text {. }
$$

The second step involves solving

$$
\begin{aligned}
&\left(\nabla^{2}+k^{2}\right) u_{2}(x, y)=\sum_{n=1}^{\infty} \sum_{m=2}^{m_{n}} c_{n m}(y) X_{n m}(x), \\
& u_{2_{x}}\left(x_{1}, y\right)=z_{1} u_{2}\left(x_{1}, y\right), u_{2_{x}}\left(x_{2}, y\right)=z_{2} u_{2}\left(x_{2}, y\right), \\
& u_{2 y}\left(x, y_{1}\right)=I_{1} u_{2}\left(x, y_{1}\right), u_{2_{y}}\left(x, y_{2}\right)=I_{2} u_{2}\left(x, y_{2}\right) .
\end{aligned}
$$

Here we substitute in the complete expansion

$$
u_{2}(x, y)=\sum_{n=1}^{\infty} \sum_{m=1}^{m_{n}} a_{n m}(y) X_{n m}(x),
$$

but we need only solve those systems corresponding to chains along which $f$ has high rank components. For we must have

$$
a_{n 1}=a_{n 2}=\cdots=a_{n m}=0
$$

for the other systems. Thus there are relatively few systems to solve. Of course, the solution to the original problem is then

$$
u=u_{1}+u_{2} \text {. }
$$

5. Extensions. This extended procedure of separation of variables, using generalized eigenfunctions, may be applied to other coordinate systems as well as to a larger class of partial differential equations. Let

$$
\xi=\xi(x, y), \quad \eta=\eta(x, y)
$$


be any conformal transformation. The Helmholtz equation then transforms to

$$
u_{\xi \xi}+u_{\eta \eta}+k^{2} h^{2}(\xi, \eta)=0 .
$$

The metrical coefficient $h^{2}$ is given by

$$
h^{2}(\xi, \eta)=x_{\xi}^{2}+x_{\eta}^{2} .
$$

A necessary and sufficient condition for separability of (5.2) with impedance boundary conditions in this system is

$$
h^{2}(\xi, \eta)=f(\xi)+g(\eta)
$$

for some $f$ and $g$ [8]. Thus all separable coordinates obtained by conformal maps of the $z=x+i y$ plane are obtained by solving

$$
x_{\xi}^{2}+x_{\eta}^{2}=f(\xi)+g(\eta) .
$$

Then $y(\xi, \eta)$ is obtained as the harmonic conjugate of $x(\xi, \eta)$. In order that the impedance conditions

$$
\frac{\partial u}{\partial n}=z(\xi, \eta) u
$$

also separate for the finite domain bounded by the coordinate curves

$$
\xi=\xi_{1}, \quad \xi=\xi_{2}, \quad \eta=\eta_{1}, \quad \eta=\eta_{2},
$$

$z(\xi, \eta)$ must be restricted to certain forms along the boundaries. By expressing $\partial u / \partial n$ in terms of $u_{\xi}$ and $u_{\eta}$ we see that $z$ must satisfy

$$
\begin{array}{ll}
z\left(\xi_{1}, \eta\right)=\frac{-C_{1}}{h\left(\xi_{1}, \eta\right)}, & z\left(\xi_{2}, \eta\right)=\frac{C_{2}}{h\left(\xi_{2}, \eta\right)}, \\
z\left(\xi, \eta_{1}\right)=\frac{-D_{1}}{h\left(\xi, \eta_{1}\right)}, & z\left(\xi, \eta_{2}\right)=\frac{D_{2}}{h\left(\xi, \eta_{2}\right)},
\end{array}
$$

where $C_{1}, C_{2}, D_{1}, D_{2}$ are complex constants. If we substitute

$$
u(\xi, \eta)=F(\xi) G(\eta)
$$

into (5.2) and (5.3) they separate into

$$
\begin{array}{lll}
F^{\prime \prime}+\left(k^{2} f-\alpha^{2}\right) F=0, & F^{\prime}\left(\xi_{1}\right)=C_{1} F\left(\xi_{1}\right), & F^{\prime}\left(\xi_{2}\right)=C_{2} F\left(\xi_{2}\right), \\
G^{\prime \prime}+\left(k^{2} g+\alpha^{2}\right) G=0, & G^{\prime}\left(\eta_{1}\right)=D_{1} G\left(\eta_{1}\right), & G^{\prime}\left(\eta_{2}\right)=D_{2} G\left(\eta_{2}\right),
\end{array}
$$

where $\alpha^{2}$ is the separation constant.

Thus, for example, for elliptic coordinates [8]

$$
f(\xi)=d^{2} \cosh ^{2} \xi, \quad g(\eta)=-d^{2} \cos ^{2} \eta,
$$

where $d$ is a real constant. Then (5.2), (5.3), and (5.5) become 


$$
\begin{gathered}
u_{\xi \xi}+u_{\eta \eta}+k^{2} d^{2}\left(\cosh ^{2} \xi-\cos ^{2} \eta\right) u=0, \\
u_{\xi}\left(\xi_{1}, \eta\right)=C_{1} u\left(\xi_{1}, \eta\right), \quad u_{\xi}\left(\xi_{2}, \eta\right)=C_{2} u\left(\xi_{2}, \eta\right), \\
u_{\eta}\left(\xi, \eta_{1}\right)=D_{1} u\left(\xi, \eta_{1}\right), \quad u_{\eta}\left(\xi, \eta_{2}\right)=D_{2} u\left(\xi, \eta_{2}\right), \\
F^{\prime \prime}+\left(k^{2} d^{2} \cosh ^{2} \xi-\alpha^{2}\right) F=0, \quad F^{\prime}\left(\xi_{1}\right)=C_{1} F\left(\xi_{1}\right), \quad F^{\prime}\left(\xi_{2}\right)=C_{2} F\left(\xi_{2}\right), \\
G^{\prime \prime}+\left(-k^{2} d^{2} \cos ^{2} \eta+\alpha^{2}\right) G=0, \quad G^{\prime}\left(\eta_{1}\right)=D_{1} G\left(\eta_{1}\right), \quad G^{\prime}\left(\eta_{2}\right)=D_{2} G\left(\eta_{2}\right) .
\end{gathered}
$$

These are Mathieu equations. We have made the assumption that the region (5.4) contains no singularities such as the origin or the focal axis.

To apply separation of variables we first determine chain basis for the $\xi$ problem. If the eigenvalues are $\alpha_{n}^{2}, n=1,2, \cdots$, and the chains are $F_{n m}(\xi)$, $F_{n m}^{+}(\xi)$ we again have

$$
\left\langle F_{n m}, F_{p q}^{+}\right\rangle=\delta_{n p} \delta_{q, m_{n}+1-m}
$$

Here $m_{n}$ is the index of $\alpha_{n}^{2}$. Substitution of

$$
u(\xi, \eta)=\sum_{n=1}^{\infty} \sum_{m=1}^{m_{n}} a_{n m}(\eta) F_{n m}(\xi), \quad r(\xi, \eta)=\sum_{n=1}^{\infty} \sum_{m=1}^{m_{n}} c_{n m}(\eta) F_{n m}(\xi)
$$

into

$$
u_{\xi \xi}+u_{\eta \eta}+k^{2} h^{2}(\xi, \eta)=r(\xi, \eta)
$$

leads to the systems

$$
\begin{array}{cc}
a_{n m_{n}}^{\prime \prime}+\left(k^{2} g+\alpha_{n}^{2}\right) a_{n m_{n}}=c_{n m_{n}}, & \\
a_{n, m_{n}-1}^{\prime \prime}+\left(k^{2} g+\alpha_{n}^{2}\right) a_{n, m_{n}-1}=c_{n, m_{n}-1}-a_{n m_{n}}, & \begin{array}{l}
a_{n m}^{\prime}\left(\eta_{1}\right)=D_{1} a_{n m}\left(\eta_{1}\right), \\
a_{n m}^{\prime}\left(\eta_{2}\right)=D_{2} a_{n m}\left(\eta_{2}\right), \\
\cdot \quad \cdot \quad
\end{array} \\
a_{n 1}^{\prime \prime}+\left(k^{2} g+\alpha_{n}^{2}\right) a_{n 1}=c_{n 1}-a_{n 2}, & \\
m=1,2, \ldots, m_{n}, \quad n=1,2, \ldots .
\end{array}
$$

If $k^{2}$ is not an eigenvalue then neither is $\alpha_{n}^{2}$, so that we may solve (5.7) by the Green's function method. If $k^{2}$ is an eigenvalue then we require $r(\xi, \eta)$ to be orthogonal to all the eigenfunctions of the adjoint of (5.2), and proceed exactly as in the $x, y$ case.

The extension to more complicated operators also involves no great changes. Thus consider the problem

$$
\left(L_{1}+L_{2}+k^{2}\right) u(x, y)=f(x, y)
$$

for a rectangle, where

$$
L_{1}=\sum_{n=0}^{p} a_{n}(x) \frac{\partial^{n}}{\partial x^{n}}, \quad L_{2}=\sum_{n=0}^{q} b_{n}(y) \frac{\partial^{n}}{\partial y^{n}} .
$$


We assume the boundary conditions on $u$ are such that substitution of $u(x, y)$ $=m(x) n(y)$ leads to the component problems

$$
\begin{aligned}
\left(L_{1}+\lambda\right) m(x) & =0, & & A m=0, \\
\left(L_{2}+\left(k^{2}-\lambda\right)\right) n(y) & =0, & & B n=0 .
\end{aligned}
$$

Here $A$ and $B$ are the usual boundary forms defined by

$$
\begin{aligned}
& A_{i} m=\sum_{j=1}^{p}\left[a_{i j} m^{(-1)}\left(x_{1}\right)+b_{i j} m^{(-1)}\left(x_{2}\right)\right], \quad i=1,2, \cdots, p, \\
& B_{i} n=\sum_{j=1}^{q}\left[c_{i j} n^{(j-1)}\left(y_{1}\right)+d_{i j} n^{(j-1)}\left(y_{2}\right)\right], \quad i=1,2, \cdots, q .
\end{aligned}
$$

Assuming the $x$ problem is regular we first find its chain bases $X_{l m n}, n=1$, $2, \cdots, r_{m l}, m=1,2, \ldots, C_{l}, l=1,2, \ldots, X_{l m n}$ is the $n$th ranked generalized eigenfunction for the $m$ th chain of the eigenvalue $\lambda_{l}$. $C_{l}$ is the number of chains for $\lambda_{l}\left(C_{l}=1\right.$ for $\left.L_{1}+L_{2}=\nabla^{2}\right)$, and $r_{m l}$ is the length of the $m$ th chain for $\lambda_{1}$. We correspondingly determine the $X_{l m n}^{+}$. The orthogonality relation is

$$
\left\langle X_{l m n}, X_{l^{\prime} m^{\prime} n^{\prime}}^{+}\right\rangle=\delta_{l l^{\prime}} \delta_{m m^{\prime}} \delta_{n, r_{m l}+1-n^{\prime}}
$$

Substituting

$$
\begin{aligned}
& u(x, y)=\sum_{l=1}^{\infty} \sum_{m=1}^{c_{l}} \sum_{n=1}^{r_{m l}} \alpha_{l m n}(y) X_{l m n}(x), \\
& f(x, y)=\sum_{l=1}^{\infty} \sum_{m=1}^{c_{l}} \sum_{n=1}^{r_{m l}} \beta_{l m n}(y) X_{l m n}(x)
\end{aligned}
$$

into (5.8), and using (5.9) and (5.10), leads to the systems

$$
\begin{gathered}
\left(L_{2}+u_{l}\right) \alpha_{l m r_{m l}}=\beta_{l m r_{m l},} \\
\left(L_{2}+u_{l}\right) \alpha_{l m, r_{m l}-1}=\beta_{l m, r_{m l}-1}-\alpha_{l m r_{m l}}, \\
\cdot \quad \cdot \quad B \alpha_{l m n}=0, \\
\cdot\left(L_{2}+u_{l}\right) \alpha_{l m 2}=\beta_{l m 2}-\alpha_{l m 3}, \\
\left(L_{2}+u_{l}\right) \alpha_{l m 1}=\beta_{l m 1}-\alpha_{l m 2}, \\
n=1,2, \cdots, r_{m l}, m=1,2, \cdots, C_{l}, l=1,2, \cdots .
\end{gathered}
$$

Here $u_{l}=k^{2}-\lambda_{l}, \beta_{l m n}(y)=\left\langle f, X_{l m, r_{m l}+1-n}^{+}\right\rangle$. If $k^{2}$ is not an eigenvalue then neither is $u_{l}$ so that (5.11) may be solved. If $k^{2}$ is an eigenvalue then we have the usual orthogonality conditions on $f(x, y)$.

Acknowledgement. The author wishes to express his gratitude to Professor Joseph B. Keller for his guidance in this work. 


\section{REFERENCES}

1. R. Courant and D. Hilbert, Methods of mathematical physics, Vol. 1, Chapter 5, Interscience, New York, 1953.

2. G. D. Birkhoff, Boundary value and expansion problems of ordinary linear differential equations, Trans. Amer. Math. Soc. 9 (1908), 373-395.

3. J. D. Tamarkin, Some general problems of the theory of ordinary linear differential equations and expansions of an arbitrary function in a series of fundamental functions, Math. Z. 27 (1927), 1-54.

4. M. A. Naimark, Investigation of the spectrum and the expansion in eigenfunctions of a nonselfadjoint differential operator of the second order on a semi-axis, Trudy Moskov. Mat. Obšč. 3 (1954), 181-270; English transl., Amer. Math. Soc. Transl. (2) 16 (1960), 103-193.

5. E. A. Coddington and N. Levinson, Theory of ordinary differential equations, Chapters 7, 11, and 12, McGraw-Hill, New York, 1955.

6. B. Friedman, Principles and techniques of applied mathematics, Chapters 4, 5, and Theorem 2.11, Applied Mathematics Series, Wiley, New York, 1956.

7. K. Knopp, Theory of functions. II, Dover, New York, 1947.

8. P. M. Morse and H. Feshbach, Methods of theoretical physics. I, Chapter 5, International Series in Pure and Applied Physics, McGraw-Hill, New York, 1953.

9. J. Schwartz, Perturbations of spectral operators and applications, Pacific J. Math. 4 (1954), 415-458.

10. M. Machover, Generalized eigenvectors and separation of variables, Doctoral Dissertation, New York Univ., New York, 1963.

11. H. P. Kramer, Perturbation of differential operators, Pacific J. Math. 7 (1957), 1405-1434.

NeW YoRK UNIVERSITY,

NeW YoRK, New York 